\title{
Atmospheric composition and structure of HD209458b
}

\author{
J.-M. Désert ${ }^{1}$, A. Vidal-Madjar ${ }^{1}$, A. Lecavelier des Etangs ${ }^{1}$, D.Sing ${ }^{1}$, \\ D. Ehrenreich ${ }^{2}$, G. Hébrard ${ }^{1}$ and R. Ferlet ${ }^{1}$ \\ ${ }^{1}$ Institut d'Astrophysique de Paris, CNRS (UMR 7095) \\ Université Pierre \& Marie Curie; 98 bis, boulevard Arago 75014 Paris, France \\ email: desert@iap.fr \\ ${ }^{2}$ Laboratoire d'Astrophysique, Observatoire de Grenoble, UJF, CNRS; \\ BP 53, F-38041 GRENOBLE Cedex 9 (France)
}

\begin{abstract}
Transiting planets like HD209458b offer a unique opportunity to scrutinize their atmospheric composition and structure. Transit spectroscopy probes the transition region between the day and night sides, called the limb. We present a re-analysis of existing HST/STIS transmission spectra of HD209458b's atmosphere. From these observations we identify H2 Rayleigh scattering, derive the absolute Sodium abundance and quantify its depletion in the upper atmosphere, extract a stratospheric T-P profile and find a temperature inversion and explain broad band absorptions with the presence of $\mathrm{TiO}$ and $\mathrm{VO}$ molecules.
\end{abstract}

\section{Introduction}

Because of the wavelength-dependent opacities of absorbing species, measurement of relative changes in eclipse depth as a function of wavelength during primary transit has the potential to reveal the presence (or absence) of specific chemical species (Seager et al. 2000a, Hubbard et al. 2001, Brown et al. 2001).

In the case of HD209458b's atmosphere, the transmission spectroscopy method led to the detection of sodium (Charbonneau et al. 2002, Sing et al. 2008b). In the UV, absorptions of several percents for H I Lyman- $\alpha$, O I and C II have been measured in the hydrodynamically escaping upper atmosphere (Vidal-Madjar et al. 2003, 2004, 2008, Désert et al. 2004, Lecavelier 2007, Ehrenreich et al. 2007).

We use public archived STIS spectra obtained during planetary transit at two spectral resolutions (low and medium). Both datasets are combined to extend the measurements over the entire optical regime to quantify possible absorbers appearing in the transmission spectrum (Sing et al. 2008a).

Here we present the identification of Rayleigh scattering by $\mathrm{H}_{2}$ molecules (Lecavelier et al. 2008b), the depletion of $\mathrm{NaI}$ as well as the extraction of a $P-T$ profile with inversion (Sing et al. 2008b). Finally, we derived the upper limits for the TiO/VO molecular abundances in the atmosphere of the planet (Désert et al. 2008).

\section{Observed features in the spectrum and interpretation}

The HST-STIS G750L, and G430L low resolution grating observations (2003) of HD209458b analyzed here are also detailed in Knutson et al. (2007a), Barman (2007), Ballester et al. (2007) and Sing et al. (2008a). For both the G750L, and G430L gratings, two visits were done for each grating, of five consecutive orbits each. Together they cover the combined range $2900-10300 \AA$, with some overlap around $5300 \AA$ with a resolving power $R=500$. 
Lecavelier Des Etangs et al. (2008a) show that the observed Absorption Depth (AD) is well approximated by:

$$
A D_{\lambda}=A D_{0}\left(1+\frac{2 H}{R_{P}} \ln \frac{\sigma_{\lambda}}{\sigma_{\lambda_{0}}}\right)
$$

where $A D_{0}$ is the depth at $\lambda=\lambda_{0}$ and $\mathrm{H}$ the scale height. Thus the observed mean absorption depth over a wavelength range of a given spectral element, is proportional to the temperature and to the logarithm of the cross section.

We use the absorption depth curve as a function of the wavelength from $4000 \AA$ to 8000 $\AA$ obtained by Sing et al. 2008a. The spectrum at wavelength below $8000 \AA$ is considered here where the absorption due to water molecules is negligible (Barman et al. 2007). This spectrum is composed of three remarkable features (see Fig. 2):

(a) Rayleigh scattering. The feature in NUV, at wavelength $\lambda \leqslant 5000 \AA$, was first reported by Ballester et al. 2007 and explained by the absorption of a hot hydrogen layer within the atmosphere. Here we propose an alternative explanation invoking the Rayleigh scattering by $\mathrm{H}_{2}$ molecules (Lecavelier et al. 2008b).

(b) Na depletion and atmospheric structure. Within the same datasets, we find that the $\mathrm{Na}$ spectral line profile is characterized by a wide absorption with a sharp transition to a narrow absorption profile at higher altitudes values (Sing et al. 2008b). This sharp transition is interpreted by condensation or ionization which deplete $\mathrm{Na}$ atoms in the upper atmosphere. Using a global fit to these data, from $3000 \AA$ to $6200 \AA$, we determine the average pressure-temperature profile $(P-T)$, see Fig. 1, at the planetary terminator (Sing et al. 2008b).

(c) $\mathrm{TiO} / \mathrm{VO}$. A third broad band absorption feature in the range $6200 \AA-8000 \AA$ appears on the absorption depth spectrum and is still unexplained. Although no typical $\mathrm{TiO}$ and VO spectral signatures have been identified unambiguously in the observed spectrum, we suggest that the opacities of those molecules are the best candidates to explain the remaining continuous broad band absorption observed in this wavelength domain (Désert et al. submitted). Using the $P$-T profile from Sing et al. $2008 \mathrm{~b}$, we derived upper limits for the $\mathrm{TiO}$ and VO abundances. We found that the abundance of $\mathrm{TiO}$ should be around $10^{-4}$ to $10^{-3}$ solar, and the abundance VO around $10^{-3}$ to $10^{-2}$ solar (Désert et al. submitted).

\section{The Irradiated Atmospheres of HD209458b}

The temperature inversion leads to high temperature at both low and high pressure. This temperature bifurcation was very well predicted by atmospheric models of strongly irradiated planets ( Hubeny et al. 2003). In the lower part of the atmosphere ( 30 mbar), the temperature is found to be in the range 1900-2400 K (Lecavelier et al. 2008b), which corresponds to the $\mathrm{M} / \mathrm{L} / \mathrm{T}$ brown dwarf regime, as expected for a hot Jupiter such as HD209458b (Kirkpatrick et al. 2005).

However, depending on the effective temperature, a large number of diatomic and polyatomic molecules are predicted to be present according to various models of brown dwarf and hot Jupiter (Burrows et al. 1999, Lodders et al. 1999, Allard et al. 2001, Lodders et al. 2002, Hubeny et al. 2003). Among those molecules and at a temperature above $1800 \mathrm{~K}$, titanium oxyde $(\mathrm{TiO})$ and vanadium oxyde (VO) in gas phase equilibrium are 


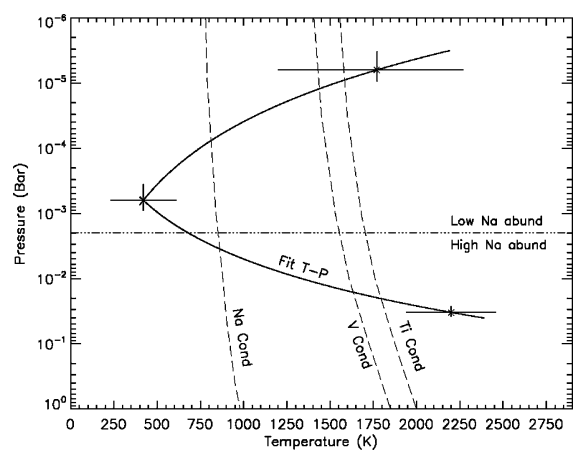

Figure 1. The atmospheric Temperature-Pressure profile ( $P-T$ profile) with error bars. The dashed lines correspond to the condensation curves for Titanium, Vanadium and Sodium. The three points of this T-P profile are derived from the fit of the observed absorption depth curve. The hot point at a pressure of 0.05 Bar is imposed by the Rayleigh scattering (See Lecavelier et al. 2008).

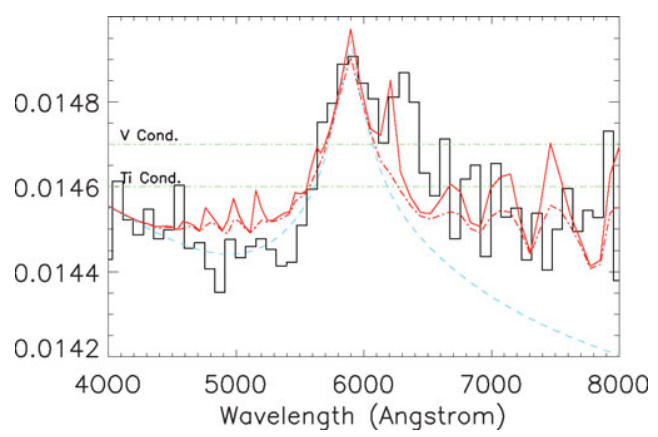

Figure 2. The low resolution STIS measurements of the planetary transit absorption depth (AD) corrected from limb-darkenning effects and binned by 60 pixels (histogram). The dashed line corresponds to the best fit model assuming Rayleigh scattering and sodium absorption with the physical T-P profile plotted in Fig. 1. Overplotted in continuous line, is the same model, with best fit models assuming Rayleigh scattering, NaI, TiO and VO absorptions. The dot-dash line correspond to the model with a cold-trap. The two altitudes of condensation for the TiO and VO molecules are plotted horizontally.

most probably present with a high abundance in strongly irradiated planet atmospheres (Seager et al. 1998, Hubeny et al. 2003, Fortney et al. 2007). Furthermore, the low albedo measurements of HD209458b (Rowe et al. 2006) rule out most of the absorbants, but TiO and VO. Recently, it has been found that a theoretical fit to the HD209458b near infrared observation at secondary eclipse requires that the dayside atmosphere of HD 209458b have a thermal inversion and a stratosphere (Knutson et al. 2008, Burrows et al. 2007c, Burrows et al. 2008). Hubeny et al. (2003) and Fortney et al. (2007) highlight the importance of gaseous $\mathrm{TiO}$ and $\mathrm{VO}$ opacity in their model of highly irradiated close-in giant planets. The last authors define two classes of irradiated atmospheres. Those which are warm enough to have a strong opacity due to $\mathrm{TiO}$ and VO gases ("pM Class" planets), and those that are cooler ("pL Class" planets) dominated by Na I and K I. Our possible detection of $\mathrm{TiO} / \mathrm{VO}$ and $\mathrm{NaI}$ in the hot atmosphere of HD209458b confirm that this planet is located in the transition region between the two classes defined by these authors.

Further observation are necessary to better characterize the two different type planets using $\mathrm{TiO} / \mathrm{VO}$. More planets can be likely studied using transit observations which 
allowed discoveries, detection, and characterization of extrasolar objects such as planets and comets (Lecavelier des Etangs et al. 1995, 1999a, 2005).

\section{References}

Allard, F., Hauschildt, P. H., Alexander, D. R., Tamanai, A., \& Schweitzer, A. 2001, ApJ, 556, 357

Ballester, G. E., Sing, D. K., \& Herbert, F. 2007, Nature, 445, 511

Barman, T. 2007, ApJ (Letters), 661, L191

Brown, T. M. 2001, ApJ, 553, 1006

Burrows, A. \& Sharp, C. M. 1999, ApJ, 512, 843

Burrows, A., Hubeny, I., Budaj, J., Knutson, H. A., \& Charbonneau, D. 2007, ApJ (Letters), 668, L171

Burrows, A., Budaj, J., \& Hubeny, I. 2008, ApJ, 678, 1436

Charbonneau, D., Brown, T. M., Noyes, R. W., \& Gilliland, R. L. 2002, ApJ, 568, 377

Charbonneau, D., Allen, L. E., Megeath, S. T., et al. 2005, ApJ, 626, 523

Désert, J.-M., Vidal-Madjar, A., Lecavelier Des Étangs, A., et al. 2004, Extrasolar Planets: Today and Tomorrow, 321, 205

Désert, J.-M., Lecavelier des Etangs, A., Vidal-Madjar, A., Sing, D. K., et al. 2008, A\&̊A, submitted

Ehrenreich, D., Lecavelier Des Etangs, A., Hébrard, G., et al. 2008, A\& A, 483, 933

Fortney, J. J., Sudarsky, D., Hubeny, I., et al. 2003, ApJ, 589, 615

Fortney, J. J., Cooper, C. S., Showman, A. P., Marley, M. S., \& Freedman, R. S. 2006, ApJ, 652,746

Fortney, J. J., Lodders, K., Marley, M. S., \& Freedman, R. S. 2008, ApJ, 678, 1419

Hubbard, W. B., Fortney, J. J., Lunine, J. I., et al. 2001, ApJ, 560, 413

Hubeny, I., Burrows, A., \& Sudarsky, D. 2003, ApJ, 594, 1011

Knutson, H. A., Charbonneau, D., Noyes, R. W., Brown, T. M., \& Gilliland, R. L. 2007, ApJ, 655,564

Knutson, H. A., Charbonneau, D., Allen, L. E., Burrows, A., \& Megeath, S. T. 2008, ApJ, 673, 526

Kirkpatrick, J. D. 2005, ARAA, 43, 195

Lecavelier Des Etangs, A., Deleuil, M., Vidal-Madjar, A., et al. 1995, A\& $A$, 299, 557

Lecavelier Des Etangs, A., Vidal-Madjar, A., \& Ferlet, R. 1999, A\&A, 343, 916

Lecavelier Des Etangs, A. 2007, A\&SA, 461, 1185

Lecavelier Des Etangs, A., Vidal-Madjar, A., Désert, J.-M., \& Sing, D. 2008, A\& A, 485, 865

Lodders, K. 1999, ApJ, 519, 793

Lodders, K. 2002, ApJ, 577, 974

Nitschelm, C., Lecavelier des Etangs, A., Vidal-Madjar, A., et al. 2000, A\&AS, 145, 275

Rowe, J. F., Matthews, J. M., Seager, S., et al. 2006, ApJ, 646, 1241

Seager, S., \& Sasselov, D. D. 1998, ApJl, 502, L157

Seager, S. \& Sasselov, D. D. 2000, ApJ, 537, 916

Sing, D. K., Vidal-Madjar, A., Desert, J.-M., Lecavelier des Etangs, A., \& Ballester, G. 2008, ArXiv e-prints, 802, arXiv:0802.3864

Sing, D. K., Vidal-Madjar, A., Lecavelier des Etangs, A., et al. 2008, ArXiv e-prints, 803, arXiv:0803.1054

Vidal-Madjar, A., Lecavelier des Etangs, A., Désert, J.-M., et al. 2003, Nature, 422, 143

Vidal-Madjar, A., Désert, J.-M., Lecavelier des Etangs, A., et al. 2004, ApJ (Letters), 604, L69

Vidal-Madjar, A., Lecavelier des Etangs, A., Désert, J.-M., et al. 2008, ApJ (Letters), 676, L57 